\title{
ANTI-WINDUP SYNTHESIS USING RICCATI EQUATIONS
}

\author{
Jorge Sofrony ${ }^{*, 1}$ Matthew C. Turner ${ }^{*, 1}$ Ian Postlethwaite ${ }^{*, 1}$ \\ * $\{j \mathrm{~s} 246, \operatorname{mct} 6, \mathrm{ixp}\}$ a le.ac.uk \\ Department of Engineering, University of Leicester, LE1 7RH, \\ $U K$.
}

\begin{abstract}
The aim of this paper is to give a novel solution to the full order anti-windup (AW) compensation problem. The main contribution of the procedure proposed is the reduction of the LMI optimisation problem that has generally been solved, to that of solving a single Riccati equation. This yields a bounded-real Riccati equation where the best performance is given by the infinity norm of the open-loop plant. This new formulation is obtained by completing the square and the synthesis method yields a compensator with two free parameters, simplifying the task of the designer to that of choosing appropriate weights. The results presented here are believed to be more intuitive, taking full adavantage of the free parameters and therefore valuable for engineering applications. Copyright IFAC 2005
\end{abstract}

Keywords: Anti-windup, Riccati equations, constrained control

\section{INTRODUCTION}

In many real life applications the control system designer encounters the problem of actuator saturation. The effects of input saturation vary from system to system, but they are commonly detrimental to the system's performance and, sometimes, stability. This problem has been tackled in many different ways, including the design of linear controllers which directly take account of the saturation (see Saberi et al. (1996) for example); model predictive control, where the control constraints are incorporated into the optimization procedure; and anti-windup (AW) and linear conditioning methods (see for example Kothare et al. (1994) and Walgama and Sternby (1993)).

Linear conditioning (or anti-windup) means augmenting the linear closed-loop with a certain linear transfer function in order to change the system's behaviour during and immediately after saturation has occurred. Linear conditioning is only intended as a precaution for systems that enter saturation occasionally and should not be implemented on systems that are outside the control constraints most of the time. AW conditioning schemes have been successful in coping with the actuator saturation problem and many different design techniques have been proposed. The standard design procedure has two steps: first, a linear controller for the nominal system without saturation is designed. Then, AW compensation is implemented to help the system during saturation periods. The AW compensator is designed in such a way that it is active only when the system undergoes saturation, modifying its behaviour and helping it return to normal linear dynamics as soon as posible. One of the main advantages of AW schemes is that linear performance is not directly restricted by the conditioning method, giving full freedom in the design of the linear controller.

\footnotetext{
1 Research supported by the UK Engineering and Physical Sciences Research Council
}

\subsection{Assumptions and Notation}

The notation used in this paper is standard throughout. The $\mathscr{L}_{p}$ norm of the time vector $y(t) \in \mathbb{R}^{n_{y}}$ is denoted as $\|y\|_{p}$ and the induced $\mathscr{L}_{p}$ norm of a nonlinear operator $\mathscr{Y}: \mathbb{Y}_{1} \rightarrow \mathbb{Y}_{2}$ from one Lebesgue space to another, as $\|\mathscr{Y}\|_{i, p}$. To avoid notational clutter the time variable $(\mathrm{t})$ and the Laplace argument ( $\mathrm{s}$ ) are omitted if no confusion is believed to arise. The Euclidean norm of the vector $y(t)$ is given by $\|y(t)\|=\sqrt{y(t)^{\prime} y(t)}$. The distance between a vector $y(t)$ and a compact set $\mathscr{Y}$ is denoted by $\operatorname{dist}(y, \mathscr{Y}):=\inf f_{\omega \in \mathscr{Y}}\|y-w\| . \mathscr{R}^{i \times j}$ represents the space of all $i \times j$ real rational transfer function matrices, and $\mathscr{R} \mathscr{H}^{\infty}$ represents the space of all real rational transfer function matrices analytic in the closed right-half plane with supremum on the imaginary axis.

\subsection{A Decoupled Scheme}

Characterising the main objective of AW compensation is subjective but the general underlying idea is simple: a fast and smooth return to linear behaviour after saturation (Kothare et al. (1994), Teel and Kapoor (1997)). We term this objective the true goal of antiwindup compensation. Although many different formulations have arisen (see for example Mulder et al. (2001) and Grimm et al. (2001)) few have been able to successfully address the true goal of AW in a general, methodical and intuitive way.

A novel method of representing most AW schemes using a single transfer function $M(s)$ was first proposed by Weston and Postlethwaite (1998). One of the main contributions of this new representation was the way in which the closed-loop compensated system could be decoupled into linear system, nonlinear loop and disturbance filter. The new decoupled structure provides a powerful tool for analysis as it defines an intuitive performance index and stability can be guaranteed using the Circle Criterion. Weston and Postlethwaite (2000) also suggested that a 
good choice of $M$ is a coprime factorization of the plant $\left(G=N M^{-1}\right)$, providing a dual representation of anti-windup compensators to that given by Kothare $e t$ al. (1994) (also used by Miyamoto and Vinnicombe (1996) and Crawshaw and Vinnicombe (2000))

Turner and Postlethwaite (2004) and Turner et al. (2004) built on the representation proposed by Weston and Postlethwaite (2000) and describe a design method that guarantees stability and performance using the $\mathscr{L}_{2}$ gain as an appropriate measure. In those papers a clear parametrisation of static, low and full order AW compensation using $M$ is given and design methods based on LMI optimisation are proposed. One of the main problems with the static and low order schemes is that there is no guarantee that one of these schemes will globally stabilise the plant in question. In contrast, there always exists a full-order anti-windup compensator which globally stabilises a linear control system with saturation, providing the plant is openloop stable. However as the full-order design method in Turner et al. (2004) is based on LMI's the procedure may suffer from numerical errors due to bad initialisation of the LMI, fast poles or large order systems. In addition, it does not take full advantage of a certain free parameter, making the design more restricted.

The main goal of this paper is to simplify this LMI formulation and obtain a new set of conditions that guarantee stability and performance, one of which is solving a Riccati equation. Although there are several commercially available LMI solvers, it is believed that the results given in this paper are an improvement, as solving Riccati equations is a standard procedure and implies less computational complexity and fewer numerical errors.

\section{NEW RESULTS}

Even though the work of Turner and Postlethwaite (2004) and Turner et al. (2004) described results where stabiltity and performance are guaranteed, the optimisation problem proposed does not take complete advantage of the free parameters available and instead these are chosen in the optimisation procedure. In Turner et al. (2003) results are given for the synthesis of full-order AW compensators, but the procedure proposed there exhibited no clear advantages over static or low-order compenstor synthesis. On the contrary, the extra states added to the closedloop compensated system makes this type of compensation unattractive. This paper will develop a new design method for full-order AW compensators based on the results given in Turner and Postlethwaite (2004). The solution here obtained reduces the computational complexity of the previous LMI formulation of Turner et al. (2004) and yields a solution where full advantage of the free design parameters is taken.

\subsection{Problem Formulation}

Consider the stabilisable, detectable and finite dimensional linear-time-invariant (FDLTI) plant $G$. The plant's dynamics subject to input saturation are given by:

$$
G(s) \sim\left\{\begin{array}{l}
\dot{x}=A x+B u_{m} \\
y=C x+D u_{m}
\end{array}\right.
$$

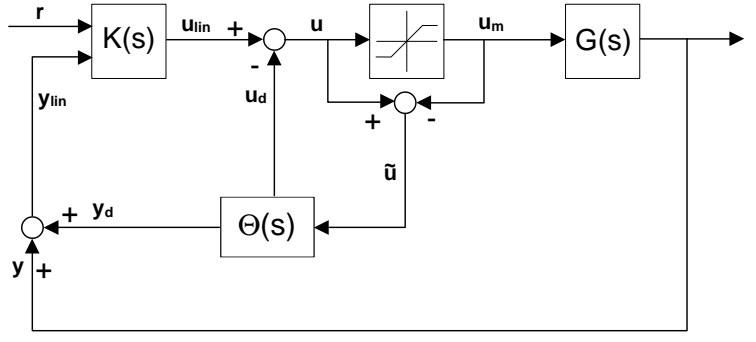

Fig. 1. Generic Anti-Windup scheme

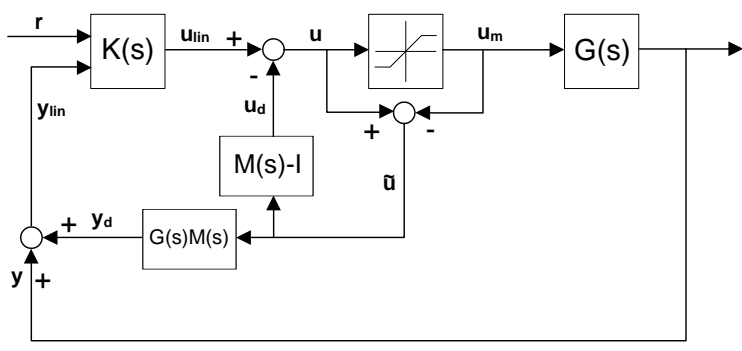

Fig. 2. Conditioning with $M(s)$

with $x \in \mathbb{R}^{n_{p}}, u_{m} \in \mathbb{R}^{m}$ and $y \in \mathbb{R}^{q}$. For simplicity disturbances are not considered although they can easily be accounted for (see Turner and Postlethwaite (2004)). In order to state global results, it is necessary to assume that the plant $G$ is stable i.e. $G(s) \in \mathscr{R} \mathscr{H}^{\infty}$. The need for this assumption will become clear later in the solution of the problem. The plant input $u_{m}$ is given by the nonlinear saturation function where:

$$
\operatorname{sat}(u)=\left[\operatorname{sat}_{1}\left(u_{1}\right), \ldots, \operatorname{sat}_{m}\left(u_{m}\right)\right]
$$

and $\operatorname{sat}_{i}\left(u_{i}\right)=\operatorname{sign}\left(u_{i}\right) \times \min \left\{\left|u_{i}\right|, \bar{u}_{i}\right\}$, where $\bar{u}_{i}>$ $0 \forall i \in\{1, \ldots, m\}$. If there is no saturation present, sat $(u)=u$, then the nominal linear closed-loop dynamics govern the system. It is also necessary to define the nonlinear deadzone function, $D z($.$) . For sim-$ plicity, define a compact set $\mathscr{U} \subset \mathbb{R}^{m}$ :

$$
\mathscr{U}:=\left[-\bar{u}_{1}, \bar{u}_{1}\right] \times \ldots \times\left[-\bar{u}_{m}, \bar{u}_{m}\right]
$$

Now define the deadzone function as:

$$
D z(u)=u-\operatorname{sat}(u)
$$

Notice that $\forall u \in \mathscr{U}, D z(u)=0$. The fact that $D z($. belongs to the Sector $[0, I]$ is of great importance for the results stated here.

Definition 1. The decentralised nonlinearity $\mathscr{N}=$ $\operatorname{diag}\left(\eta_{1}, \ldots, \eta_{m}\right)$ is said to belong to Sector $[0, I]$ if the following inequality holds:

$$
\eta_{1}\left(u_{i}\right)^{2} \leq \eta_{i}\left(u_{i}\right) u_{i} \leq u_{i}^{2} \quad \forall i \in\{1, \ldots, m\}
$$

This definition will later allow us to pose an $\mathscr{H}_{\infty^{-}}$ type optimization problem formulation of the Circle Criterion.

Figure 1 shows a generic anti-windup configuration, where $G(s)$ is the plant described earlier and $K(s)$ is the controller which has been designed to stabilise the nominal (un-saturated) plant and achieve some nominal performance specifications. $\Theta(s)$ is the antiwindup compensator which only becomes active once saturation has occurred. The compensator has two sets of outputs, $\theta_{1}=u_{d} \in \mathbb{R}^{m}$ and $\theta_{2}=y_{d} \in \mathbb{R}^{q}$, which enter the control input and the plant output respectively. 


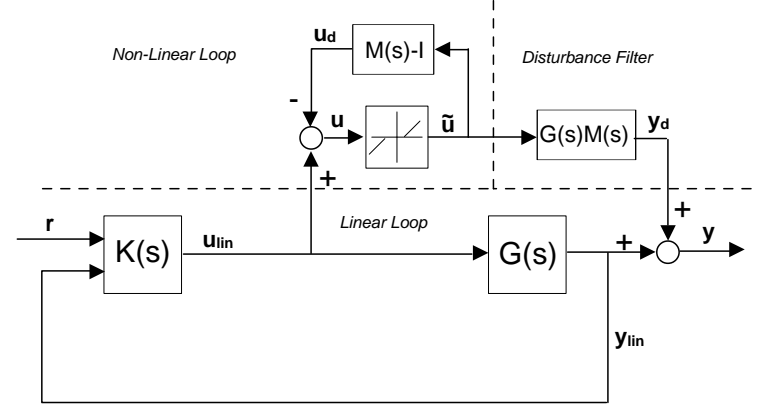

Fig. 3. Decoupled structure

The anti-windup formulation of Weston and Postlethwaite (2000) is depicted in Figure 2 where the antiwindup compensator $\Theta(s)$ has been paramtrised by the transfer function $M(s)$. It is also possible, keeping all signal labels the same and using identity (2), to transform Figure 2 into Figure 3. This new representation is significant as there is a clear decoupling of the closeloop compensated system into nominal linear system, nonlinear loop and disturbance filter.

Observing the decoupled system in Figure 3 it is clear that the mapping $\mathscr{T}: u_{\text {lin }} \mapsto y_{d}$ determines, in some sense, the deviation of the nonlinear system from the nominal linear system. It therefore follows that it is desirable to maintain $\|\mathscr{T}\|_{i, p}$ small in some appropriate $\mathscr{L}_{p}$ norm. Since the performance index is purely defined on the saturated system, no constraints are set directly on the controller $K$. This means that for an appropriate choice of $M$ it is possible to ignore $K$. The only consideration made is that $K$ has been previously designed for good performance and stability of the nominal linear plant. The following definition will formally describe the AW problem to be considered

Definition 2. The anti-windup compensator parmetrised by $M(s)$, is said to solve the anti-windup problem if the closed loop system in Figure 2 (equivalently Figure 3) is internally stable and well-posed and the following hold:

(1) If $\operatorname{dist}\left(u_{\text {lin }}, \mathscr{U}\right)=0, \quad \forall t \geq 0$, then $y_{d}=0, \quad \forall t \geq$ 0 (assuming zero initial conditions for $M$ ).

(2) If $\operatorname{dist}\left(u_{\text {lin }}, \mathscr{U}\right) \in \mathscr{L}_{p}$, then $y_{d} \in \mathscr{L}_{p}$ for some integer $p \in[1, \infty)$.

The transfer function $M$ is said to solve strongly the anti-windup problem if, in addition, the following condition is satisfied:

(3) The operator $\mathscr{T}: u_{\text {lin }} \mapsto y_{d}$ is well-defined and finite gain $\mathscr{L}_{p}$ stable for some integer $p \in[1, \infty)$.

\subsection{Representing $M(s)$}

The transfer function $M$ in Figure 2 has to capture the original setup proposed by the designer (fullorder, static, sub-optimal). In this paper only full order AW compensators are studied. Observe how $y_{d}$ is the system's deviation from linear dynamics, making it desirable to maintain it small. This means shaping the disturbance filter frequency response in order to reject the effects of the nonlinear input $\tilde{u}$. However, since $M$ is common for both the nonlinear loop and the disturbance filter, trade-offs must be made when choosing it. Notice that making $M$ 'small' implies that the output from $G M$ will be 'small', meaning good performance. Also notice that this would make the term $M-I \approx-I$, meaning that the nonlinear loop is close to being ill-posed.

In order for lightly damped and slow poles of $G$ not to appear in the disturbance filter they must somehow be cancelled by $M$. This suggests that a good choice of $M$ would be a right coprime factorisation of the plant, $G=N M^{-1}$. Notice how the disturbance filter reduces to $N$ and the AW compensator is independent of the controller $K$. It was shown in Weston and Postlethwaite (2000) that a suitable representation for $M$ and $N$, without introducing any extra states, is:

$$
\left[\begin{array}{l}
\Theta_{1} \\
\Theta_{2}
\end{array}\right]=\left[\begin{array}{c}
M-I \\
N
\end{array}\right]=\left\{\begin{array}{l}
\dot{x}=(A+B F) x+B \tilde{u} \\
u_{d}=F x \\
y_{d}=(C+D F) x+D \tilde{u}
\end{array}\right.
$$

where $F$ is a free parameter and $A+B F$ must be Hurwitz.

\subsection{Stability and Performance Analysis}

The problem of stability and performance is addressed by minimizing $\mathscr{T}: u_{\text {lin }} \mapsto y_{d}$ for a certain $\mathscr{L}_{p}$ norm. For the case of $p=2$, the problem can be expressed as that of finding the minimum $\gamma>0$ such that $\|\mathscr{T}\|_{i, 2} \leq$ $\gamma$. The following procedure not only allows the synthesis of an optimal compensator, but also ensures stability and gives a measure of global performance if the plant $G$ is assumed asymptotically stable. It is now possible to present sufficient conditions which ensure a finite $\mathscr{L}_{2}$ performance gain of the AW compensator.

Theorem 3. There exists a full order anti-windup compensator $\Theta=\left[\begin{array}{ll}\Theta_{1}^{\prime} & \Theta_{2}^{\prime}\end{array}\right]^{\prime} \in \mathscr{R}^{(m+q) \times m}$, as stated in (4), which solves strongly the anti-windup problem for the case of $p=2$ if there exist matrices $P=P^{T}>0$, $W=\operatorname{diag}\left(\omega_{1}, \ldots, \omega_{m}\right)>0$ and a postive real scalar $\gamma$ such that the following Ricatti equation is satisfied

$$
\tilde{A}^{\prime} P+P \tilde{A}+P B R^{-1} B^{\prime} P+\tilde{Q}=0
$$

where

$$
\begin{aligned}
& \tilde{A}=A+B R^{-1} D^{\prime} C \\
& \tilde{Q}=C^{\prime}\left(I+D R^{-1} D^{\prime}\right) C \\
& R=\left(\gamma^{2} I+D^{\prime} D\right)>0
\end{aligned}
$$

and

$$
Z=\left(2 W-D^{\prime} D-\gamma^{-2} W^{2}\right)>0
$$

Furthermore, if equation (5) is satisfied, a suitable $\Theta$ achieving $\|\mathscr{T}\|_{i, 2}<\gamma$ is obtained by calculating the matrix gain $F$ as follows:

$$
F=-\gamma^{2} R^{-1}\left(W^{-1}-\gamma^{-2}\right)\left(B^{\prime} P+D^{\prime} C\right)
$$

Proof: Notice that in order to solve strongly the AW compensation problem, it is necessary to meet the conditions stated in Definition 2. It is easy to observe that the first two conditons are trivially met if internal stability of the closed-loop compensated system is guaranteed, assuming zero initial conditions for the AW compensator. As will be shown later, by choosing $F$ as described in Theorem 1, it is possible to guarantee that $\|\mathscr{T}\|_{i, 2}<\gamma$ for any $\gamma>\|G\|_{\infty}$, therefore solving strongly the AW compensation problem.

For algebraic simplicity, consider the case where $D=$ 
0 (the proof when $D \neq 0$ involves much more algebra and hence for space reasons is omitted). Note that as $D z(.) \in \operatorname{Sector}[0, \mathrm{I}]$,

$$
\tilde{u}_{i} u_{i} \geq \tilde{u}_{i}^{2}, \quad \forall i \in\{1, \ldots, m\}
$$

From this it follows that for some matrix $W=$ $\operatorname{diag}\left(w_{1}, \ldots, w_{m}\right)>0$

$$
\tilde{u}^{\prime} W(u-\tilde{u}) \geq 0
$$

Next assume $\exists v(x)=x^{\prime} P x>0$, then if

$$
\begin{aligned}
L\left(x, u_{\text {lin }}, \tilde{u}, F, W\right) & :=\frac{d}{d t} x^{\prime} P x+\left\|y_{d}\right\|^{2}-\gamma^{2}\left\|u_{l i n}\right\|^{2} \\
& +\tilde{u}^{\prime} W(u-\tilde{u})
\end{aligned}
$$

is negative definite, it follows that $\dot{v}(x)<0$ is a Lyapunov inequality and the closed loop system is stable. Also notice that if $L\left(x, u_{l i n}, \tilde{u}, F, W\right)<0$, integrating $L$ from 0 to $\mathrm{T}$ and taking the limit when $T \rightarrow \infty$ yields $\left\|y_{d}\right\|_{2}<\gamma\left\|u_{\text {lin }}\right\|_{2}$ and hence $\|\mathscr{T}\|_{i, 2}<\gamma$. To summarise, if equation (13) is negative definite, the anti-windup problem is solved in the $\mathscr{L}_{2}$ sense. Evaluating equation (13) results in an inequality containing several cross terms in $\tilde{u}, u_{\text {lin }}$, and $x$. In order to overcome this problem (if an LMI formulation is to be avoided) it is necessary to complete the square several times.

Definition 4. Completing the square refers to the fact that

$$
\left(\alpha x-\alpha^{-1} y\right)^{\prime}\left(\alpha x-\alpha^{-1} y\right)=\alpha^{2} x^{\prime} x+\alpha^{-2} y^{\prime} y-x^{\prime} y-y^{\prime} x
$$

so that cross-product terms can be expressed as a difference between squares.

Expanding (13) and substituting $u=u_{\text {lin }}-u_{d}$ gives

$$
\begin{gathered}
L=x^{\prime} C^{\prime} C x-\gamma^{2} u_{l i n}^{\prime} u_{l i n}+\dot{x}^{\prime} P x+x^{\prime} P \dot{x}-\tilde{u}^{\prime} W u_{d}-u_{d}^{\prime} W \tilde{u} \\
-2 \tilde{u}^{\prime} W \tilde{u}+\tilde{u}^{\prime} W u_{l i n}+u_{\text {lin }}^{\prime} W \tilde{u}<0
\end{gathered}
$$

We now eliminate the cross-product terms in three steps.

(I) Cross-product terms involving $u_{\text {lin }}$ and $\tilde{u}$ are grouped as follows:

$$
\begin{gathered}
-\left[\gamma^{2} u_{\text {lin }}^{\prime} u_{\text {lin }}-\tilde{u}^{\prime} W u_{\text {lin }}-u_{\text {lin }}^{\prime} W \tilde{u}\right]=-\left(\gamma u_{\text {lin }}-\right. \\
\left.\gamma^{-1} W \tilde{u}\right)^{\prime}\left(\gamma u_{\text {lin }}-\gamma^{-1} W \tilde{u}\right)+\gamma^{-2} \tilde{u}^{\prime} W^{2} \tilde{u}
\end{gathered}
$$

Combining the above with (14), a cost function containing no cross-product terms between $u_{\text {lin }}$ and $\tilde{u}$ is obtained. Using equation (4) to expand $\dot{x}$ and noticing that $u_{d}=F x$ :

$$
\begin{gathered}
L=x^{\prime}\left(C^{\prime} C+A^{\prime} P+P A+F^{\prime} B^{\prime} P+P B F\right) x+\tilde{u}^{\prime}\left(B^{\prime} P-W F\right) x+ \\
x^{\prime}\left(P B-F^{\prime} W\right) \tilde{u}-\tilde{u}^{\prime}\left(2 W-\gamma^{-2} W^{2}\right) \tilde{u}-\left(\gamma u_{\text {lin }}-\right. \\
\left.\gamma^{-1} W \tilde{u}\right)^{\prime}\left(\gamma u_{\text {lin }}-\gamma^{-1} W \tilde{u}\right) \leq 0
\end{gathered}
$$

(II) Cross-Product terms involving $\tilde{u}$ and $x$ are grouped, including the extra term $\gamma^{-2} \tilde{u}^{\prime} W^{2} \tilde{u}$ from (I), as follows:

$$
\begin{aligned}
& -\left[\tilde{u}^{\prime}\left(2 W-\gamma^{-2} W^{2}\right) \tilde{u}-\tilde{u}^{\prime}\left(B^{\prime} P-W F\right) x-x^{\prime}\left(B^{\prime} P-W F\right)^{\prime} \tilde{u}\right] \\
& =-\left(Z^{\frac{1}{2}} \tilde{u}-Z^{-\frac{1}{2}}\left(B^{\prime} P-W F\right) x\right)^{\prime}\left(Z^{\frac{1}{2}} \tilde{u}-Z^{-\frac{1}{2}}\left(B^{\prime} P-\right.\right. \\
& W F) x)+x^{\prime}\left(B^{\prime} P-W F\right)^{\prime} Z^{-1}\left(B^{\prime} P-W F\right) x
\end{aligned}
$$

Note that $Z=\left(2 W-\gamma^{-2} W^{2}\right)$ must be positive definite in order to have a well-posed problem. This condition arises from the necessity of making the term
$\left(Z^{\frac{1}{2}} \tilde{u}-Z^{-\frac{1}{2}}\left(B^{\prime} P-W F\right) x\right)^{\prime}\left(Z^{\frac{1}{2}} \tilde{u}-Z^{-\frac{1}{2}}\left(B^{\prime} P-W F\right) x\right)$ negative for any pair $(\tilde{u}, x)$. It can easily be shown that if $Z \leq 0$, this is not always guaranteed. By replacing this new group of terms, a new cost function is obtained. This cost function has no cross-product terms between $\tilde{u}$ and $x$.

$$
\begin{gathered}
L=x^{\prime}\left(C^{\prime} C+A^{\prime} P+P A+F^{\prime} B^{\prime} P+P B F+P B Z^{-1} B^{\prime} P-\right. \\
\left.P B Z^{-1} W F-F^{\prime} W Z^{-1} B^{\prime} P+F^{\prime} W Z^{-1} W F\right) x \\
-\left(Z^{\frac{1}{2}} \tilde{u}-Z^{-\frac{1}{2}}\left(B^{\prime} P-W F\right) x\right)^{\prime}\left(Z^{\frac{1}{2}} \tilde{u}-Z^{-\frac{1}{2}}\left(B^{\prime} P-\right.\right. \\
W F) x)-\left(\gamma u_{\text {lin }}-\gamma^{-1} W \tilde{u}\right)^{\prime}\left(\gamma u_{\text {lin }}-\gamma^{-1} W \tilde{u}\right) \leq 0
\end{gathered}
$$

(III) Terms involving $F$ and $F^{\prime} F$ are grouped: $F^{\prime} W Z^{-1} W F-\left(W Z^{-1} B^{\prime} P-B^{\prime} P\right)^{\prime} F-F^{\prime}\left(W Z^{-1} B^{\prime} P-B^{\prime} P\right)=$ $\left(Z^{\frac{-1}{2}} W F-Z^{\frac{1}{2}} W^{-1}\left(W Z^{-1}-I\right) B^{\prime} P\right)^{\prime}\left(Z^{\frac{-1}{2}} W F-Z^{\frac{1}{2}} W^{-1}\left(W Z^{-1}-\right.\right.$ I) $\left.B^{\prime} P\right)-P B\left(W Z^{-1}-I\right)^{\prime} W^{-1} Z W^{-1}\left(W Z^{-1}-I\right) B^{\prime} P$

This last step will yield an expression for the matrix gain $F$.

Finally, by using the results given in (III) we obtain an expression for our cost function (13) as

$$
L\left(x, u_{\text {lin }}, \tilde{u}, F, W\right)=L_{a}+L_{b}+L_{c}
$$

where

$$
\begin{aligned}
L_{a}= & x^{\prime}\left(C^{\prime} C+A^{\prime} P+P A+P B Z^{-1} B^{\prime} P-\right. \\
P B & \left.\left(W Z^{-1}-I\right)^{\prime} W^{-1} Z W^{-1}\left(W Z^{-1}-I\right) B^{\prime} P\right) x \\
L_{b}= & +x^{\prime}\left(Z^{-\frac{1}{2}} W F-Z^{\frac{1}{2}} W^{-1}\left(W Z^{-1}-I\right) B^{\prime} P\right)^{\prime} \times \\
& \left(Z^{-\frac{1}{2}} W F-Z^{\frac{1}{2}} W^{-1}\left(W Z^{-1}-I\right) B^{\prime} P\right) x \\
L_{c}= & -\left(Z^{\frac{1}{2}} \tilde{u}-Z^{-\frac{1}{2}}\left(B^{\prime} P-W F\right) x\right)^{\prime} \times \\
& \left(Z^{\frac{1}{2}} \tilde{u}-Z^{-\frac{1}{2}}\left(B^{\prime} P-W F\right) x\right) \\
& -\left(\gamma u_{\text {lin }}-\gamma^{-1} W \tilde{u}\right)^{\prime}\left(\gamma u_{\text {lin }}-\gamma^{-1} W \tilde{u}\right)
\end{aligned}
$$

Equation (16) is comprised of three terms. The last term $L_{c}$ is a negative definite quadratic term. Therefore if the first two terms can be set to zero, then $L()<$.0 . Setting the second term, $L_{b}$, to zero yields a condition for the gain matrix $F$.

$$
\begin{gathered}
\left(Z^{-\frac{1}{2}} W F-Z^{\frac{1}{2}} W^{-1}\left(W Z^{-1}-I\right) B^{\prime} P\right)=0 \\
F=\left(\gamma^{-2}-W^{-1}\right) B^{\prime} P
\end{gathered}
$$

where $P=P^{T}>0$ comes from solving the Ricatti equation which makes the first term $L_{a}=0$ :

$$
\begin{aligned}
& C^{\prime} C+A^{\prime} P+P A+P B Z^{-1} B^{\prime} P- \\
& P B\left(W Z^{-1}-I\right)^{\prime} W^{-1} Z W^{-1}\left(W Z^{-1}-I\right) B^{\prime} P=0
\end{aligned}
$$

which, after some algebraic manipulation, reduces to:

$$
C^{\prime} C+A^{\prime} P+P A+\gamma^{-2} P B B^{\prime} P=0
$$

These are exactly the conditions given in the theorem (with $D_{p}=0$ ). Internal stability guarantees part (1) of the anti-windup problem is satisfied; the finite $\mathscr{L}_{2}$ gain of $\mathscr{T}$ ensures part (3) is satisfied, and hence part (2) is also satisfied. Well-posedness of the loop is guaranteed by the lack of direct feedthrough terms i.e. $M-I$ is strictly proper. This shows that the strong AW problem has been solved

Remark 1: Notice that the Riccati equation given is of bounded-real type and only has a solution if $G(s)$ is stable and for any $\gamma$ such that $\|G\|_{\infty}=\gamma_{o p t} \leq \gamma$. That is, 


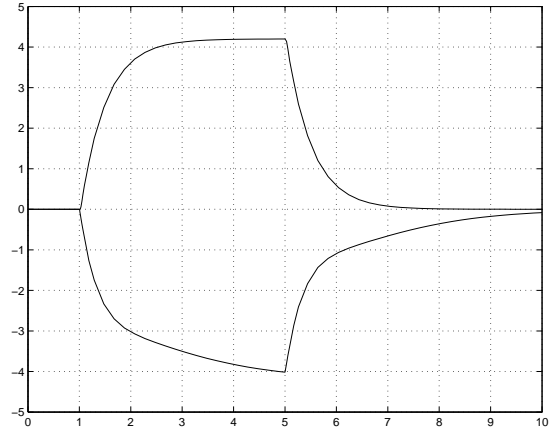

Fig. 4. Output response of missile (nominal linear)

the performance level of the anti-windup compensator is given by the $\mathscr{H}_{\infty}$ norm of the open-loop plant. This suggests that optimal anti-windup performance is obtained when $\gamma=\gamma_{o p t}$, leaving the designer the task of choosing $W>0$. This freedom in choosing $W$ is absent in Turner et al. (2004) and (Mulder et al. 2001) and hence we have recovered freedom in choosing the so-called stability multiplier.

\section{EXAMPLE}

In this section the effectiveness of the results are shown through an example from the literature. The example is that of a missile auto-pilot introduced by Rodriguez and Cloutier (1994) and used by Turner and Postlethwaite (2004) and Romanchuk (1999). The plant is a simplified model of the dynamics of the rollyaw channels of a bank-to-turn misile.

$$
\begin{aligned}
A_{p} & =\left[\begin{array}{ccc}
-0.818 & -0.999 & 0.349 \\
80.29 & -0.579 & 0.009 \\
-2734 & 0.05621 & -2.10
\end{array}\right], \\
B_{p} & =\left[\begin{array}{cc}
0.147 & 0.012 \\
-194.4 & 37.61 \\
-2716 & -1093
\end{array}\right], \quad B_{p d}=\left[\begin{array}{ll}
0 & 0 \\
0 & 0 \\
0 & 0
\end{array}\right] \\
C_{p} & =\left[\begin{array}{lll}
1 & 0 & 0 \\
0 & 1 & 0
\end{array}\right], \quad D_{p}=D_{p d}=\left[\begin{array}{ll}
0 & 0 \\
0 & 0
\end{array}\right]
\end{aligned}
$$

A nominal linear LQG/LTR controller yields excellent nominal closed loop time and frequency responses and is given by

$$
\left[\begin{array}{c|c|c}
A_{c} & B_{c} & B_{c r} \\
\hline C_{c} & D_{c} & D_{c r}
\end{array}\right]=\left[\begin{array}{cc|c|c}
A_{c 1} & B_{c 1} & 0 & 0 \\
0 & 0 & -I & I \\
\hline C_{c 1} & 0 & 0 & 0
\end{array}\right]
$$

where

$$
\begin{aligned}
& A_{c 1}=\left[\begin{array}{ccccc}
-0.29 & -107.8 & 6.67 & -2.58 & -0.4 \\
107.68 & -97.81 & 63.95 & -4.52 & -5.35 \\
-6.72 & 64.82 & -54.19 & -40.79 & 5.11 \\
3.21 & 2.1 & 29.56 & -631.15 & 429.89 \\
0.36 & -3.39 & 3.09 & -460.03 & -0.74
\end{array}\right] \\
& B_{c 1}=\left[\begin{array}{cc}
2.28 & 0.48 \\
-40.75 & 2.13 \\
18.47 & -0.22 \\
-2.07 & -44.68 \\
-0.98 & -1.18
\end{array}\right] \\
& C_{c 1}=\left[\begin{array}{ccccc}
0.86 & 8.54 & -1.71 & 43.91 & 1.12 \\
2.17 & 39.91 & -18.39 & -8.51 & 1.03
\end{array}\right]
\end{aligned}
$$

The actuators have saturation limits of \pm 8 in both channels

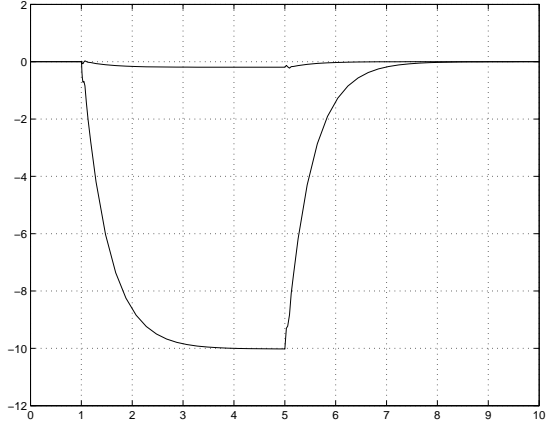

Fig. 5. Control response of missile (nominal linear)

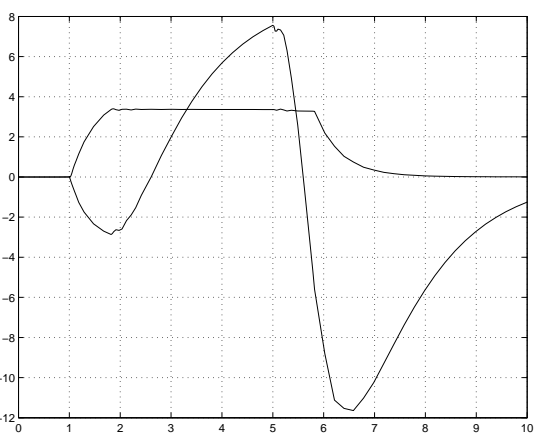

Fig. 6. Output response of missile (saturation, no AW)

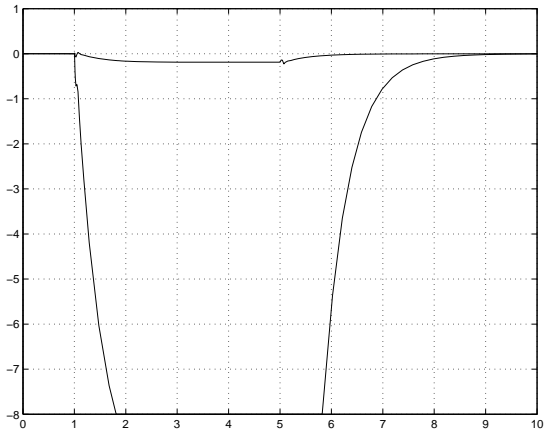

Fig. 7. Control response of missile (saturation, no AW)

Figures 4 and 5 show the nominal linear response of the missile for a pulse $r=[4.2-4.2]$. Notice the system's excellent response and decoupled outputs. However, observe how the control signal is outside the set $\mathscr{U}=\operatorname{Co}\{(8,8),(-8,8),(-8,-8),(8,-8)\}$ for some time. This suggests that the system with saturated actuators might have poor performance and may even be unstable. Figures 6 and 7 confirm this and show clearly how the system loses its decoupling properties.

Figure 8 shows the missile response with the full order AW compensation proposed in this paper. Notice the improvement over the uncompensated response: the saturated system now tends to follow the linear response closely and the return to nominal linear dynamics is swift. Notice how, after saturation i.e. $u-$ $\operatorname{sat}(u)=0$, the system displays some additional dynamics introduced by the AW compensator. This suggests that the AW compensator must have fast and well damped poles.

Figure 9 shows the output response of the missile when the reference amplitude has been increased to 10 in the first channel and a 3-2-1 type demand in both channels. Notice that without AW, tracking ability is 


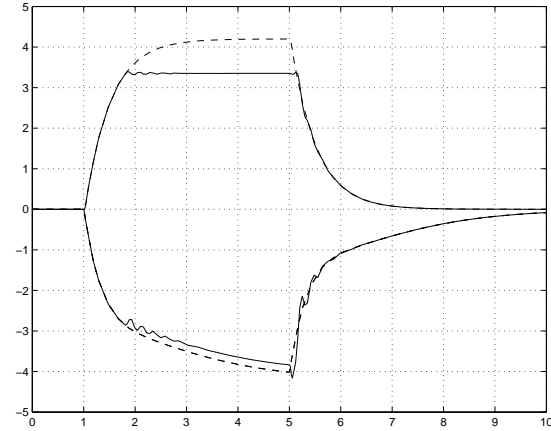

Fig. 8. Output response of missile: - saturation and full-order AW; - - nominal linear

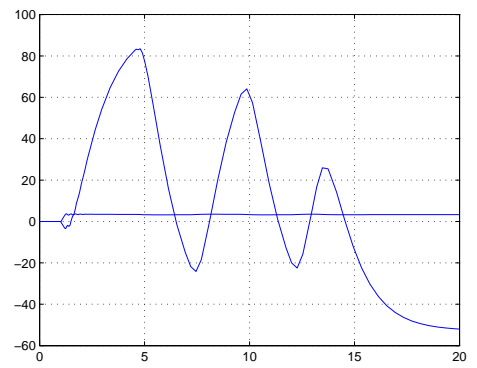

Fig. 9. Output response of missile for 3-2-1 type input: saturation and no anti-windup

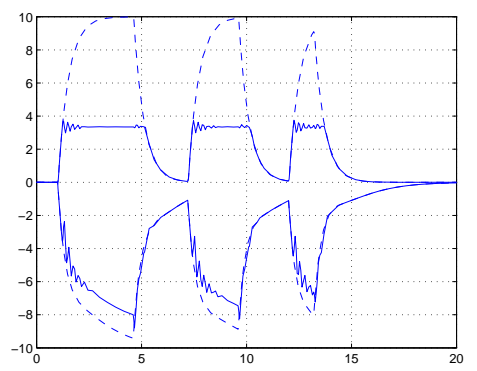

Fig. 10. Output response of missile for 3-2-1 type input: - saturation and full-order AW; - - nominal linear

completely lost and stability is barely maintained. Figure 10 shows the performance improvement which is obtained using the suggested compensator: although the tracking objectives are unobtainable, the qualititative response of the system remains close to that of the linear system.

From (21) and the real bounded lemma it is possible to conclude that $\gamma \geq\|G(s)\|_{\infty}=\gamma_{\text {opt }}$. This suggests that optimal performance is obtained when $\gamma=\gamma_{o p t}$, leaving the designer with the task of choosing $W$. Observe from equation (4) that $M(s)$ is a function of $F$, and consequently the size of the control signal $u_{d}$ and the compensator dynamics are affected by it. The poles of the AW compensator, i.e. eig $(A+B F)$, are a function of $F$ and therefore increasing the size of $F$ (decreasing $W$ ), increases the control signal $u_{d}$ and yields a compensator with faster dynamics.

Having fast poles is of no concern in continuous time aplications. However, in simulation and implementation, time is discretised and the sampling rate becomes of great importance. If the poles of the AW com- pensator are faster than the sampling rate, numerical errors are likely. In this case, $W$ can be chosen large in order to reduce the size of the poles and avoid possible numerical errors introduced when discretising.

\section{CONCLUSIONS}

This paper has presented an alternative solution to the full-order AW problem. The solution given is novel in the sense that most other full-order AW design techniques which ensure stability and performance involve LMI's (see Grimm et al. (2001) for a general treatment): here we simply require the solution to a bounded real type of Riccati equation. The solution is also believed to be more intuitive because the free parameter, $W>0$ is clearly linked to the poles of the anti-windup compensator, which has important practical relevance.

Further work may consider reducing the number of states of 'full order' AW compensators, which are not always implementable in problems with a large number of states. A possible way of obtaining a reduced order compensator may include using conventional model reduction methods on the nominal plant before using the design process proposed in this paper. This implies that the existing method must be modified so as to take account of the 'uncertainties' introduced when reducing the model.

\section{REFERENCES}

Crawshaw, S. and G. Vinnicombe (2000). Anti-windup synthesis for guaranteed $\mathscr{L}_{2}$ performance. Proc. IEEE Conf. on Decision and Control.

Grimm, G., I. Postlethwaite, A.R. Teel, M.C. Turner and L. Zaccarian (2001). Linear matrix inequalities for full and reduced order anti-windup synthesis. Proc. American Control Conf.

Kothare, M.V., P.J. Campo, M. Morari and C.N. Nett (1994). A unifi ed framework for the study of anti-windup designs. Automatica 30(12), 1869-1883.

Miyamoto, S. and G. Vinnicombe (1996). Robust control of plants with saturation nonlinearity based on comprime factor representations. Proc. IEEE Conf. on Decision and Control pp. 2838-2840.

Mulder, E.F., M.V. Kothare and M. Morari (2001). Multivariable anti-windup controller synthesis using linear matrix inequalities. Automatica 37(9), 1407-1416.

Rodriguez, A.A and J. R. Cloutier (1994). Control of a bank-toturn missile with saturating actuators. Proc. American Control Conference.

Romanchuk, B.G. (1999). Some comments on anti-windup synthesis using LMI's. International Journal of Robust and Nonlinear Control 9(10), 717-734.

Saberi, A., Z. Lin and A.R. Teel (1996). Control of linear systems with saturating actuators. IEEE Trans. on Automatic Control 41(3), 368-378.

Teel, A.R. and N. Kapoor (1997). The $\mathscr{L}_{2}$ anti-windup problem: Its defi nition and solution. Proc. European Cont. Conf.

Turner, M.C. and I. Postlethwaite (2004). A new perspective on static and low order anti-windup synthesis. International Journal of Control 77(1), 27-44.

Turner, M.C., G. Herrmann and I. Postlethwaite (2003). Discretetime anti-windup: Part 1 - stability and performance. Proc. European Control Conference.

Turner, M.C., G. Herrmann and I. Postlethwaite (2004). Accounting for robustness in anti-windup synthesis. Proc. American Control Conference.

Walgama, K.S. and J. Sternby (1993). Conditioning technique for multiinput multioutput processes with input saturation. IEE Proceedings D 140(4), 231-241.

Weston, P.F. and I Postlethwaite (1998). Analysis and design of linear conditioning schemes for systems containing saturating actuators. IFAC Nonlinear Control System Design Symposium.

Weston, P.F. and I Postlethwaite (2000). Linear conditioning for systems containing saturating actuators. Automatica 36(9), 13471354. 